\title{
Voting Analysis in Political Science
}

\author{
Chang Bum Kim
}

Department of Mathematics, Kookmin University, Seoul 136-702, South Korea.

\begin{abstract}
In this paper we consider voting analysis in the political science in connection with $B_{n}$ (or $M_{n}\{0,1\}$ ), the semigroup of the binary relations on $X$ with $n$ elements. We also consider it in connection with $M_{n}(F)$ (or $B_{n}(F)$ ), the semigroup of all fuzzy binary relations on $X$. Also we establish a possibility theorem and an impossibility theorem in voting analysis based on preferences in $B_{n}$ and $M_{n}(F)$.
\end{abstract}

Key words : fuzzy preference, rational relation, fuzzy binary relation, semigroup.

\section{Introduction}

K. J. Arrow[1] introduced the social choice function based on the rational relations. Here the rational relation $R$ is a relation satisfying the following conditions:

1. $R$ is connected ( $\Leftrightarrow$ for all $x, y \in P, x R y$ or $y R x$ ),

2. $R$ is transitive ( $\Leftrightarrow$ for all $x, y, z \in P, x R y$ and $y R z$ imply $x R z)$.

Also he studied the possibility theorem and impossibility theorem for social welfare functions.

J. B. Kim[5] investigated the fuzzy rational choice functions, and C. B. Kim[4] studied $B_{n}\left(\right.$ or $\left.M_{n}\{0,1\}\right)$, the semigroup of the binary relations on $X$ with $n$ elements.

Recently in journals there are papers concerning possibility-theorem and impossibility-theorem (See [3, 6, $7,8])$.

In particular, S. Zahariev[8] considered the correction between the impossibility theorem of Arrow and group choice with fuzzy preference relations.

In this paper, we consider voting analysis in the political science in connection with rational relations and also we establish a possibility-theorem and an impossibilitytheorem in voting analysis based on preferences in $B_{n}$ and $M_{n}\{0,1\}$ (or $M_{n}(F)$ ), the semigroup of the binary relations on $X$ with $n$ elements. (A preference in $M_{n}(F)$ will be called a fuzzy preference.)

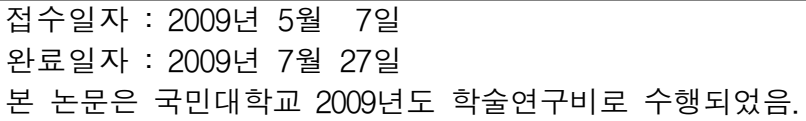

\section{Preliminaries}

In this section, we consider several definitions and theorems.

Definition 2-1. Let $X$ be a set of $n$ distinct elements. Let $R$ be a subset of $X \times X$, and if $(a, b) \in R$, then we often write $a R b$. We denote the set of all such $R$ by $B_{n}$.

Definition 2-2. If $R \in B_{n}$, then we can have a matrix representation $M(R)$ of $R$ : If $X$ is a finite set, $X=$ $\left\{x_{1}, x_{2}, \ldots, x_{n}\right\}$, then we define $M(R)=A=\left(a_{i j}\right)$ as follows : $a_{i j}=1$, if $\left(x_{i}, x_{j}\right) \in R$ and $a_{i j}=0$, if $\left(x_{i}, x_{j}\right) \notin$ $R$. We call $B_{n}$ the semigroup of all binary relations $R$ on $X$. Define $M_{n}\{0,1\}=\left\{M(R)=A=\left(a_{i j}\right): R \in B_{n}\right\}$.

Theorem 2-3. $B_{n}$ is isomorphic to $M_{n}\{0,1\}$.

Proof. It is easy.

Definition 2-4. $M_{n}\{0,1\}$ of Theorem 2-3 is called the Boolean matrix semigroup of order $n$ over 0 and 1 .

Definition 2-5 ([4]). Let $R$ be an element of $B_{n}$, the semigroup of all binary relations on $X$ with $n$ elements. Let $[0,1]$ be the unit interval of the real line. If there exists a function $f_{R}: X \times X \longrightarrow[0,1]$ such that $f_{R}(x, y)=$ $t \in(0,1]$ if $(x, y) \in R$ and $f_{R}(x, y)=0$ if $(x, y) \notin R$, then we say that $\left(R, f_{R}\right)$ is a a fuzzy relation on $X$. We denote the set of all fuzzy relations on $X$ by $B_{n}(F)$. Let $\left(R, f_{R}\right) \in B_{n}(F)$. Then we have a matrix representation of $\left(R, f_{R}\right)$ by ; Let $M\left(R, f_{R}\right)=A=\left(a_{i j}\right)$. Then $a_{i j}=t$, if $f_{R}\left(x_{i}, x_{j}\right)=t \in[0,1]$. 
Note. Let $F=[0,1]$. If $a, b \in F$, we define $a+b=$ $\max \{a, b\}$ and $a b=\min \{a, b\}$.

Definition 2-6 ([4]). Let $M_{n}([0,1])=\left\{M\left(R, f_{R}\right)=A=\right.$ $\left.\left(a_{i j}\right):\left(R, f_{R}\right) \in B_{n}(F)\right\}$ and define $A B=C=\left(c_{i j}\right)$ as $c_{i j}=\sum_{k=1}^{n} a_{i k} b_{k j}$, for $A=\left(a_{i j}\right)$ and $B=\left(b_{i j}\right)$ in $M_{n}([0,1])$.

Theorem 2-7. $M_{n}(F)$ forms a semigroup under the product operation given in Definition 2-6.

Proof. It is obvious.

We call $M_{n}(F)$ the semigroup of full fuzzy matrices over $[0,1]$. And we call $B_{n}(F)$ the semigroup of all fuzzy relations on $X$ with $n$ elements.

Suppose there is a city (say, MOON-CITY) with the population $N(>1)$ at a time $t(=$ April 3, 2009). The MOON-CITY is going to have new Mayor election on a day (July 5, 2009) to choose new Mayor from $m$ candidates $p_{i} \in P=\left\{p_{1}, p_{2}, \ldots, p_{m}\right\}$ by voting. Every one $i$ of the city residents has the right to vote with preference $R_{i}$ as a subset $P \times P$, and we consider $R_{i}$ as a member of $B_{m}$, the semigroup of the binary relations on $P$. If $i$ will vote for $p_{j}=x$ on the election day, then we write $R_{i}(x)=R_{i}\left(p_{j}\right)=x$.

We define $R(x)=\left\{i: 1 \leq i \leq N\right.$ and $\left.R_{i}(x)=x\right\}$. If there exists $x_{0} \in P$ such that $|R(y)| \leq\left|R\left(x_{0}\right)\right|$ for all $y$ in $P$ and at least one $y_{0} \in P$ such that $\left|R\left(y_{0}\right)\right|<\left|R\left(x_{0}\right)\right|$, then we say that $x_{0}$ will be elected as new Mayor on the election day. We summarize this as the following theorem.

Theorem 2-8. $x_{0} \in P$ is elected if and only if $\left|R\left(x_{0}\right)\right| \geq$ $|R(y)|$ for all $y$ in $P$ and if there exists $y_{0}$ in $P$ such that $\left|R\left(y_{0}\right)\right|<\left|R\left(x_{0}\right)\right|$.

We define the relation $R$ on $P$ as follows : $x R y$ if and only if $|R(y)| \leq|R(x)|$. Then we have the following theorem.

Theorem 2-9. The relation $R$ satisfies the above conditions 1 and 2, i.e., the relation $R$ is rational.

Proof. Clearly, always either $|R(y)| \leq|R(x)|$ or $|R(x)| \leq$ $|R(y)|$, so that for all $x$ and $y, x R y$ or $y R x$. Thus $R$ is connect. To show transitivity, suppose $x R y$ and $y R z$. Then $|R(y)| \leq|R(x)|$ and $|R(z)| \leq|R(y)|$. Thus $|R(z)| \leq$ $|R(x)|$ and thus $x R z$. Hence $R$ is transitive. Therefore the relation $R$ is rational.

\section{Main Results}

In this section we consider voting analysis by fuzzy relations.

Example 3-1 ([4]). The MOON-CITY has five persons (denoted by $R=\{x, y, z, u, v\}$ ) and two mayor candidates $P=\{x, y\}$. For $t \in R$, we denote the preference by a $2 \times 2$ fuzzy matrix,

$$
R_{t}=\left(\begin{array}{ll}
t_{11} & t_{12} \\
t_{21} & t_{22}
\end{array}\right) .
$$

We define $R_{t}(x)<R_{t}(y)$ if and only if $t_{11}<t_{22}$ and say that $t$ will vote for $y$ instead of $x$. Define $R(x)=\{t \in$ $\left.R: R_{t}(y)<R_{t}(x)\right\}$ and $R(y)=\left\{t \in R: R_{t}(x)<\right.$ $\left.R_{t}(y)\right\} . \quad x$ is winner by Number if $|R(y)|<|R(x)|$. Also we define $\|R(x)\|=x_{11}+y_{11}+z_{11}+u_{11}+$ $v_{11}$ and $\|R(y)\|=\sum_{t \in R} t_{22}$. We shall say that $x$ is winner by Quality if and only if $\|R(x)\|>\|R(y)\|$. Let

$$
\begin{gathered}
R_{x}=\left(\begin{array}{ll}
1 & 0 \\
0 & 0
\end{array}\right), \quad R_{y}=\left(\begin{array}{ll}
0 & 0 \\
0 & 1
\end{array}\right), R_{z}=\left(\begin{array}{ll}
0.6 & 0.3 \\
0.6 & 0.5
\end{array}\right) \\
R_{u}=\left(\begin{array}{ll}
0.1 & 0.2 \\
0.1 & 0.8
\end{array}\right), \text { and } R_{v}=\left(\begin{array}{ll}
0.6 & 0.6 \\
0.4 & 0.5
\end{array}\right)
\end{gathered}
$$

Then we see that $|R(x)|=3,|R(y)|=2,\|R(x)\|=$ $2.3,\|R(y)\|=2.8$ and we can say that $x$ is the winner by Number but $y$ is the winner by Quality. Let

$$
\begin{aligned}
R_{1}(x) & =R_{x} R_{z} R_{v} \\
& =\left(\begin{array}{ll}
1 & 0 \\
0 & 0
\end{array}\right)\left(\begin{array}{ll}
0.6 & 0.3 \\
0.6 & 0.5
\end{array}\right)\left(\begin{array}{ll}
0.6 & 0.6 \\
0.4 & 0.5
\end{array}\right) \\
& =\left(\begin{array}{cc}
0.6 & 0.6 \\
0 & 0
\end{array}\right)
\end{aligned}
$$

as party preference of three members $x, z$, and $v$. Similarly let $R_{2}(y)=R_{y} R_{u}=\left(\begin{array}{cc}0.0 & 0.0 \\ 0.1 & 0.8\end{array}\right)$ and we say that $y$ is the winner by two parties vote because $R_{2}(y)$ has 0.8 at $(2,2)$ entry and $R_{1}(x)$ has 0.6 at $(1,1)$ entry.

Definition 3-2. Let $x$ be a candidate in the $P$. $x$ is said to be the perfect winner if $|R(y)|<|R(x)|$ for $y \neq x$ and if $\|R(y)\|<\|R(x)\|$.

We have a possibility theorem as follows:

Theorem 3-3. Let $P=\{x, y, \ldots\}$ be the set of candidates for the Mayor election of the MOON-CITY whose population is $N(>2)$. Then it is possible for $x$ in $P$ to win as the perfect winner in the Mayor election.

Proof. It is possible for $x$ to have $|R(y)|<$ $|R(x)|$ and $\|R(y)\|<\|R(x)\|$, if $N>2$.

Definition 3-4. A candidate $x$ in $P$ is said to be the purely perfect winner, if $\frac{\|R(x)\|}{N}=1$, where $N$ is the population of the city. 
Theorem 3-5 (AN IMPOSSIBILITY THEOREM). Any candidate $x$ can not be the purely perfect winner in the Mayor election. (We assume that the number of candidates is greater than 1).

Proof. If there are candidates more than one, then any candidate $x$ has an opposite candidate $y(\neq x)$. Thus the fuzzy preference $R_{y}$ has $y_{11}=0$, which shows that $\frac{\|R(x)\|}{N}<1$, where $N$ is the population. This completes the proof.

\section{Acknowledgements}

I express my thanks to the referees for their valuable comments and suggestions.

\section{References}

[1] K. J. Arrow, Social Choice and Individual Values, New Haven and London, Yale University Press (1951).

[2] A. Banerjee, Fuzzy Choice Functions, Revealed Preference and Rationality, Fuzzy Sets and Systems 70 (1995), 31-43.

[3] C. Hägg, Possibility and Cost in Decision Analysis, Fuzzy Sets and Systems 1 (1978), 81-86.
[4] C. B. Kim, A Study of the Semigroup $B_{n}$ of the Binary Relations on a Set $X$ with $n$ Elements, Ph.D. Thesis, Yonsei University, (1985).

[5] J. B. Kim, Fuzzy Rational Choice Functions, Fuzzy Sets and Systems 10 (1983), 37-43.

[6] G. Păun, An Impossibility Theorem for Indicators Aggregation, Fuzzy Sets and Systems 9 (1983), $205-$ 210 .

[7] T. Tanino, Fuzzy Preference Orderings in Group Decision Making, Fuzzy Sets and Systems 12 (1984), 117-131.

[8] S. Zahariev, An Approach to Group Choice with Fuzzy preference Relations, Fuzzy Sets and Systems 22 (1987), 203-213.

\section{Chang Bum Kim}

Professor of Kookmin University

Research Area : Fuzzy Mathematics, Fuzzy Algebra

E-mail : cbkim@kookmin.ac.kr 